\begin{tabular}{|c|c|c|c|}
\hline \multirow{2}{*}{$\begin{array}{r}\text { Case Reports in } \\
\text { Gastroenterology }\end{array}$} & \multicolumn{2}{|c|}{ Case Rep Gastroenterol 2017;11:377-381 } & \multirow[b]{2}{*}{$\begin{array}{l}\text { Karger } \\
\text { Open access }\end{array}$} \\
\hline & $\begin{array}{l}\text { DOI: 10.1159/000475917 } \\
\text { Published online: June 14, } 2017\end{array}$ & $\begin{array}{l}\text { (c) } 2017 \text { The Author(s) } \\
\text { Published by S. Karger AG, Basel } \\
\text { www.karger.com/crg }\end{array}$ & \\
\hline & $\begin{array}{l}\text { This article is licensed under } \\
\text { International License (CC BY- }- \\
\text { Usage and distribution for comn }\end{array}$ & $\begin{array}{l}\text { nons Attribution-NonCommercia } \\
\text { ger.com/Services/OpenAccessLice } \\
\text { uires written permission. }\end{array}$ & \\
\hline
\end{tabular}

\title{
A Complicated Thread: Abdominal Actinomycosis in a Young Woman with Crohn Disease
}

\author{
Ari Nahum Gregory Filice Ashish Malhotra \\ Minneapolis Veterans Hospital, Minneapolis, MN, USA
}

\section{Keywords}

Abdominal actinomycosis · Crohn disease · Actinomyces · Actinomycosis · Inflammatory bowel disease

\begin{abstract}
Crohn disease is a chronic inflammatory condition that primarily affects the gastrointestinal tract. Typical manifestations include fever, weight loss, fatigue, and abdominal pain, and abdominal abscesses and fistulae are frequent complications. Abdominal actinomycosis is a subacute or indolent disease associated with Actinomyces spp. Symptoms can be very similar to those of Crohn disease, and fistulae are also common. Since ulcerations in the intestinal tract are thought to be caused by Actinomyces escaping from the gut lumen and establishing intra-abdominal infection, it seems likely that abdominal actinomycosis may occur in patients with inflammatory bowel disease. We report a case of abdominal actinomycosis in a woman with active Crohn disease.

(C) 2017 The Author(s)

Published by S. Karger AG, Basel
\end{abstract}

\section{Case Report}

A 31-year-old woman with Crohn disease presented in March 2014 with 3 weeks of worsening abdominal pain and fevers [1]. The pain was primarily in the right lower quadrant, waxing and waning in nature, and described as "cramping." She denied diarrhea, melena, nausea, vomiting, or hematochezia. She had been having night sweats but denied any 
weight loss. Two weeks prior to hospitalization, a gastroenterologist had prescribed $40 \mathrm{mg}$ of prednisone daily for a possible Crohn flare, but her symptoms did not improve.

She had been diagnosed with Crohn disease in 2007, and phenotypically she had fistulizing Crohn disease complicated by terminal ileitis and a perianal fistula. She had a complicated course due to poor compliance with medical regimens and had been maintained mostly on intermittent oral steroids. In addition to her history of Crohn disease, she had an intrauterine birth control device (IUD) placed in 2010, and a root canal treatment was performed on a molar tooth 3 months prior to admission.

On initial presentation, physical exam showed moderate right lower quadrant tenderness. Laboratory analysis was significant for a total leukocyte count of 17,000 cells/ $\mu \mathrm{L}$ with $84 \%$ neutrophils. A CT of the abdomen showed inflammation of the terminal ileum with a fluid collection of $1.0 \mathrm{~cm}$ in diameter between the ileum and uterus. Intravenous piperacillintazobactam ( $3.375 \mathrm{~g}$ every $6 \mathrm{~h}$ ) was started, and oral prednisone was continued.

Two days later, the abdominal fluid collection was drained percutaneously. Cultures of the fluid yielded 4+ Streptococcus anginosus and 2+ of an unidentified gram-negative bacillus. Symptoms improved over the next 3 days, and she was discharged with a 14-day course of oral ciprofloxacin (500 mg twice daily) and metronidazole (500 mg 3 times daily) plus ongoing prednisone therapy.

In May 2014, the patient acutely developed worsening lower abdominal pain, fevers, and diarrhea. On presentation, her temperature was $102.4^{\circ} \mathrm{F}$, and she had bilateral lower quadrant abdominal tenderness. Laboratory analysis showed a total leukocyte count of 24,000 cells/ $\mu \mathrm{L}$ with $81 \%$ neutrophils. An abdominal MRI (Fig. 1) demonstrated severe terminal ileitis with strictures and 4 complex rim-enhancing fluid collections in the lower abdominal and pelvic cavity. Several fistulae tracked from the ileum to the abscesses and to a separate loop of the small bowel. One of the fluid collections had eroded through the fascial planes of the abdominal wall.

The patient received intravenous piperacillin/tazobactam (3.375 g every $6 \mathrm{~h}$ ), and a percutaneous drain was placed into one of the fluid collections. Cultures yielded heavy growth of Actinomyces odontolyticus and moderate growth of Fusobacterium spp. Two days later, repeat CT of the abdomen showed residual fluid collections and a second drainage catheter was placed. Fluid cultures from that specimen grew 2+A. odontolyticus and 2+ Staphylococcus lugdunensis. Pelvic examination was normal with no evidence of endometritis, and her IUD was removed.

The patient received piperacillin/tazobactam for 2 weeks and was discharged on oral penicillin VK (1 g 4 times daily) plus probenecid (500 $\mathrm{mg}$ twice daily). By the time of discharge, her fever and diarrhea had resolved and her abdominal pain had improved. Two weeks later, azathioprine (100 mg orally daily) plus adalimumab (40 mg subcutaneously every 2 weeks) were started. Prednisone doses were tapered over the next 2 months and then stopped. After a follow-up abdominal CT and a sonogram had confirmed that the abscesses and fistulae had fully collapsed, the drains were removed.

In August 2014, the patient's penicillin dose was decreased to $250 \mathrm{mg} 4$ times daily because of mild nausea which subsequently improved. The patient felt well except for mild loose stools, which were typical of her Crohn disease. Then, in April 2015, she developed new abdominal pain. An abdominal CT revealed an intra-abdominal fluid collection where the previous abscesses had been. Percutaneous drainage yielded $35 \mathrm{~mL}$ of purulent fluid. A specimen for culture yielded rare Enterobacter cloacae and moderate growth of $A$. odontolyticus. Ciprofloxacin (750 mg orally twice daily) was added, and penicillin and probenecid were continued. 
In May 2015, the patient developed lower abdominal pressure at the end of micturition and 2 weeks later she developed fevers. An abdominal CT scan and a sonogram revealed 2 pelvic abscesses, the largest of which was $3.3 \mathrm{~cm}$, with a fistulae to the bladder. The fluid collections were drained and cultures yielded Candida glabrata, C. dubliniensis, S. epidermidis, and lactobacillus. She received intravenous piperacillin/tazobactam initially and then was discharged on oral amoxicillin (1 mg twice daily), moxifloxacin (400 mg daily), and fluconazole (800 mg daily).

In August 2015, moxifloxacin and fluconazole were discontinued and amoxicillin was continued. Aside from mild diarrhea, which had been present for years and was attributed to Crohn disease, she felt well.

\section{Discussion}

Actinomyces are anaerobic or microaerophilic gram-positive bacilli that inhabit the human oral cavity, primarily the gingival crevices. A. israelii is the most common species associated with human disease, but several other Actinomyces spp. can inhabit the same niche and are associated with this disease [2-4]. Actinomyces spp. are swallowed and sometimes isolated from gut contents, but they are usually transient [4]. Actinomyces spp. also colonize the genital tract in some women with foreign bodies in the vagina or uterus, most commonly IUDs [5]. Endometritis develops in a minority of colonized patients, with occasional spread to the pelvic organs and abdominal cavity $[6,7]$.

The most common actinomycosis syndromes include cervicofacial infection, pneumonia, and mediastinitis $[3,4,8]$. Abdominal actinomycosis is a less common and represents a distinct clinical syndrome [9]. It typically occurs after Actinomyces escape from the gut into other abdominal organs [9] or the peritoneal cavity or occurs by extension of a uterine infection in women with IUDs [5]. In actinomycosis, infections frequently erode and cross tissue planes and fistulae can develop. With abdominal disease, fistulae may be present between organs or coursing exteriorly through the abdominal wall or perineum [9].

We found only 3 published cases of abdominal actinomycosis mimicking inflammatory bowel disease [10-12] and only 1 case of abdominal actinomycosis in a patient with known inflammatory bowel disease [13]. In our case, the fact that 3 abscess cultures over nearly 1 year yielded $A$. odontolyticus is strong evidence that $A$. odontolyticus was involved in the pathogenesis of this patient's abscesses. Since the patient had severe Crohn disease with recurrent ileitis, it seems likely that a defect in her bowel mucosa allowed $A$. odontolyticus to establish a sinus tract and eventually an abscess. Although the patient had received prednisone, neither short- nor long-term prednisone use has been recognized as a risk factor for developing actinomycosis.

Poor dentition, dental carries, and dental procedures are all established risk factors for cervicofacial actinomycosis $[2,14]$, although there is no literature regarding their role in abdominal actinomycosis. Interestingly, given the timing of the patient's root canal treatment relative to her initial presentation, it seems possible that infected oral material released by the dental procedure passed down the gut and escaped into the peritoneal cavity.

The presence of an IUD in our patient's cervix, near the abdominal abscesses, raised the possibility that actinomycosis developed initially within the uterus and then spread to the lower abdomen. However, this seemed unlikely as the abdominal CT images showed no abnormalities of the uterus, and the cervical and uterine mucosae appeared healthy according to a gynecologist at the time of IUD removal. 
Actinomycosis is diagnosed by the observation of Actinomyces spp. in tissues or isolation of Actinomyces spp. from a site of disease that could not have been contaminated with mucosal flora. Preferred treatment is with penicillin for 6-12 months. For penicillin-allergic patients, alternatives include doxycycline, ceftriaxone, clindamycin, and erythromycin [3].

This case strongly suggests that abdominal actinomycosis can occur as a complication of Crohn disease and should be considered in patients with Crohn disease who present with intra-abdominal fluid collections. Subacute or chronic abdominal pain with fever or leukocytosis, a history of recent dental treatments, and evidence of an infectious process that erodes fascial planes or forms fistulae are all suggestive of actinomycosis. We recommend that specimens of fistulous secretions or abscesses be submitted for anaerobic culture in cases of Crohn disease with these complications and that physicians request that specimens submitted for culture be processed in ways that will maximize isolation and identification of Actinomyces spp.

\section{Statement of Ethics}

The authors have no ethical disclosures.

\section{Disclosure Statement}

The authors have no financial disclosures.

\section{References}

Baumgart DC, Sandborn WJ: Crohn's disease. Lancet 2012;380:1590-1605.

Boyanova L, Kolarov R, Mateva L, et al: Actinomycosis: a frequently forgotten disease. Future Microbiol 2015;10:613-628.

3 Valour F, Senechal A, Dupieux C, et al: Actinomycosis: etiology, clinical features, diagnosis, treatment, and management. Infect Drug Resist 2014;7:183-197.

4 Smego RA Jr, Foglia G: Actinomycosis. Clin Infect Dis 1998;26:1255-1261; quiz 1262-1263.

5 Westhoff C: IUDs and colonization or infection with Actinomyces. Contraception 2007;75:S48-S50.

-6 Evans DT: Actinomyces israelii in the female genital tract: a review. Genitourin Med 1993;69:54-59.

7 Choi MM, Baek JH, Lee JN, et al: Clinical features of abdominopelvic actinomycosis: report of twenty cases and literature review. Yonsei Med J 2009;50:555-559.

8 Wong VK, Turmezei TD, Weston VC: Actinomycosis. BMJ 2011;343:d6099. inflammatory bowel disease. Acta Gastroenterol Belg 2001;64:318-320.

11 Sevilla Chica F, Villalba Ferrer F, Domingo Del Pozo C, et al: Abdominal actinomycosis simulating Crohn's disease. Gastroenterol Hepatol 2001;24:300-302.

12 Acevedo F, Baudrand R, Letelier LM, et al: Actinomycosis: a great pretender. Case reports of unusual presentations and a review of the literature. Int J Infect Dis 2008;12:358-362.

13 Manley PN, Dhru R: Actinomycosis complicating Crohn's disease. Gastroenterology 1980;79:934-937.

14 de Andrade AL, Novaes MM, Germano AR, et al: Acute primary actinomycosis involving the hard palate of a diabetic patient. J Oral Maxillofac Surg 2014;72:537-541. 


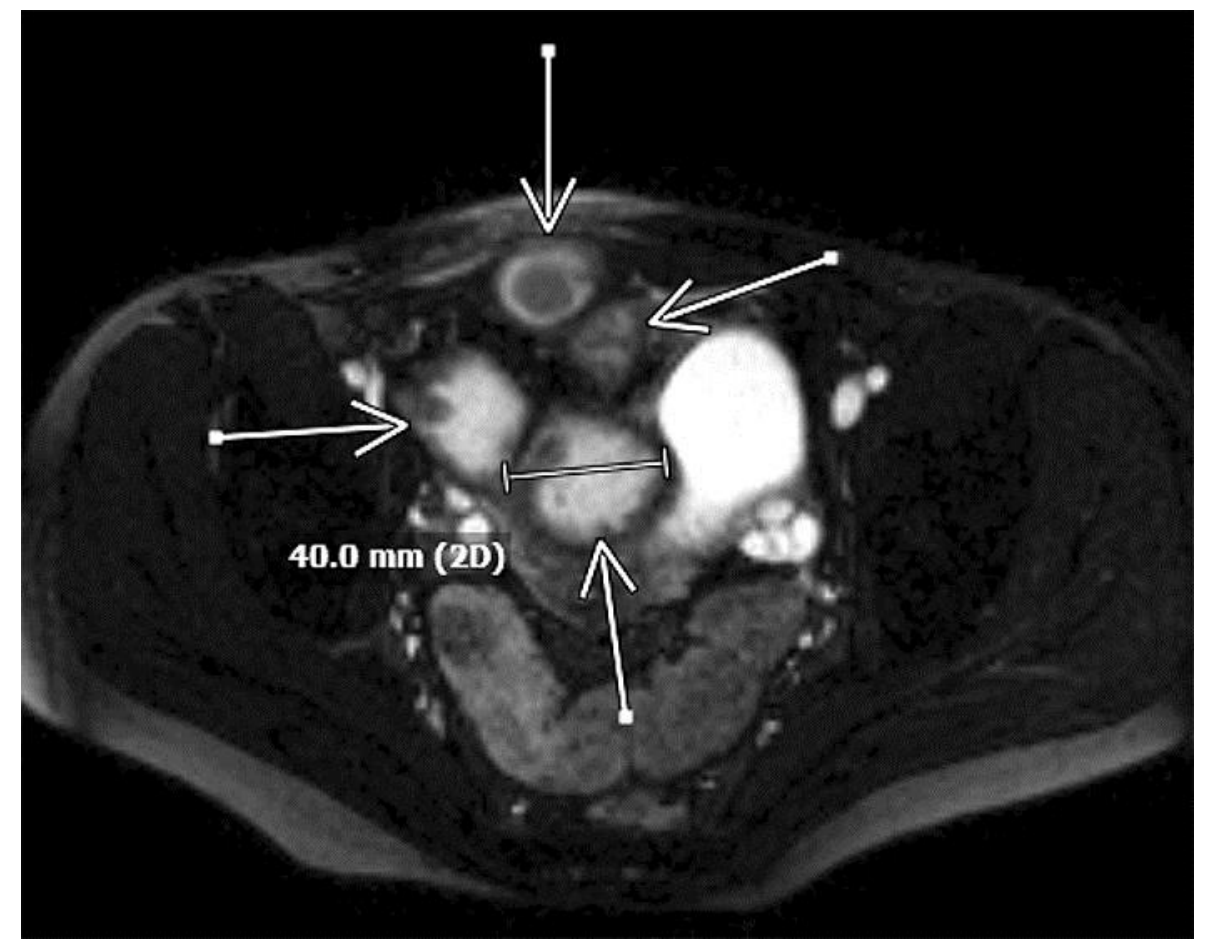

Fig. 1. MRI of the abdomen and pelvis showing 4 fluid collections, the largest of which is $4 \mathrm{~cm}$ in diameter. The most superficial abscess is eroding into the fascial planes of the peritoneum with associated soft tissue edema of abdominal wall.

Nahum et al.: A Complicated Thread: Abdominal Actinomycosis in a Young Woman with Crohn Disease 